\title{
Diagnostic Instrumentation System for the SRRC 1.3 GeV Synchrotron Radiation Light Source
}

\author{
K. T. Hsu, G. J. Jan*, C. H. Kuo and K. H. Hu \\ Synchrotron Radiation Research Center \\ Hsinchu 30077, Taiwan, R. O. C. \\ * Department of Electrical Engineering, National Taiwan University \\ Taipei 10764, Taiwan, R. O. C.
}

\begin{abstract}
Many types of diagnostic instruments, such as beam position monitors, fluorescence screen monitors, intensity monitors, stripline electrodes and synchrotron radiation monitor system, ...etc., were designed and built into the beam transport line (BTL) and storage ring (SR) to facilitate and enable commissioning and operation. The VME crate based data acquisition and processing as well as timing control electronic module were designed and developed that is quite useful for machine commissioning and operation. The beam instruments have been installed and tested on the BTL and SR. The test and characterization results in SRRC synchrotron radiation facilities will be summarized in the paper.
\end{abstract}

\section{INTRODUCTION}

The nearly completed $1.3 \mathrm{GeV}$ synchrotron radiation facilities at SRRC is composed of a full energy injector, a 70 $\mathrm{m}$ long transport line and a $120 \mathrm{~m}$ long storage ring [1]. Various diagnostics devices are needs for all stages of commissioning and routine operation of the transport line and the storage ring [2]. Most of the diagnostics devices fulfil his role during the commissioning phase despite some diagnostics devices still need some adjustment.

\section{STORAGE RING DIAGNOSTICS}

Beam position are measured by 47 beam position monitors. Averaged beam intensity are measured by direct current current transformer. Fast beam structure are observed by fast current transformer. Two linear tapered striplines are used to measure transverse and longitudinal oscillation as well as time structure. One excitation electrode is used to excitate betatron oscillation or used to damp transverse oscillation. Screen monitors are used to aid the turning of the first turn. Transvesre beam profile can be measured from synchrotron radiation light directly.

\section{A. Beam position monitoring system}

The signals picked up by four button electrodes was processed arithmetically to obtain the horizontal and vertical position as well as total intensity of the electron beam. The processing electronics are shown in Figure 1 [3]. The PIN diode RF multiplexer are used to select the signal pick-up by the button electrode, single channel heterodyne receiver to detect the electron beam signal. The detected signal by processing electronic are read into VME crate. Beam position information are updated into the dynamic database on process

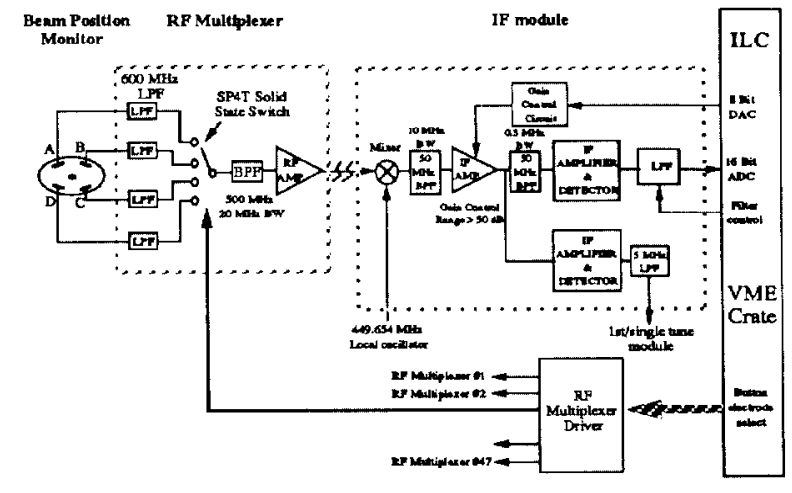

Figure 1. Block diagram of the BPM processing electronics

computer and workstation periodically with rate about 10 times per second. The BPM electronics can be worked when beam current as small as $50 \mu \mathrm{A}(-70 \mathrm{dBm})$ with resolution about $50 \mu \mathrm{m}$ and as large as $200 \mathrm{~mA}(-10 \mathrm{dBm})$ with $10 \mu \mathrm{m}$ resolution. From the statistics of 1000 measured value with constant signal input as shown in Figure 2, the figure show that resolution of the BPM are better than $10 \mu \mathrm{m}$ when the beam current large than several mA. Beam current dependence of the beam position measured are shown in Figure 3, the test result reveal that the drift versus the beam current does not exceed a $20 \mu \mathrm{m}$ peak-to-peak amplitude over 1 to 200 inA range when the beam at center. The orbit measurement speed is software selectable, 200 reading per second can be achieved easily if necessary.
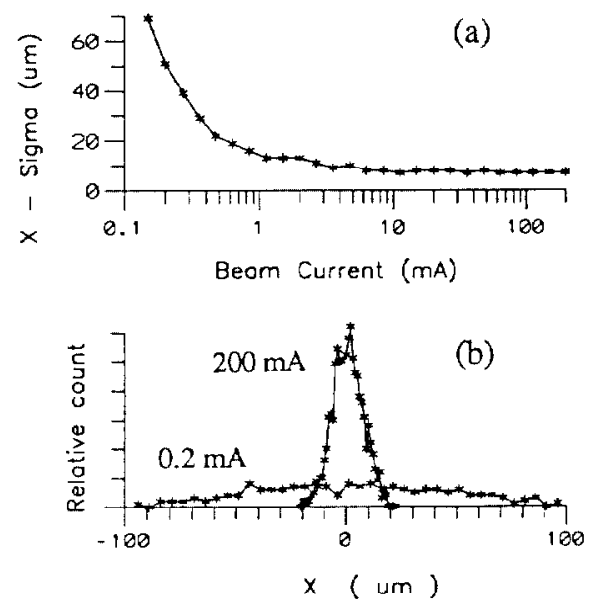

Figure 2. Performance test by using simulate signal (a) Resolution versus beam current of the BPM system, (b) Statistical distribution of 1000 beam position reading for beam current 0.2 and $200 \mathrm{~mA}$. 


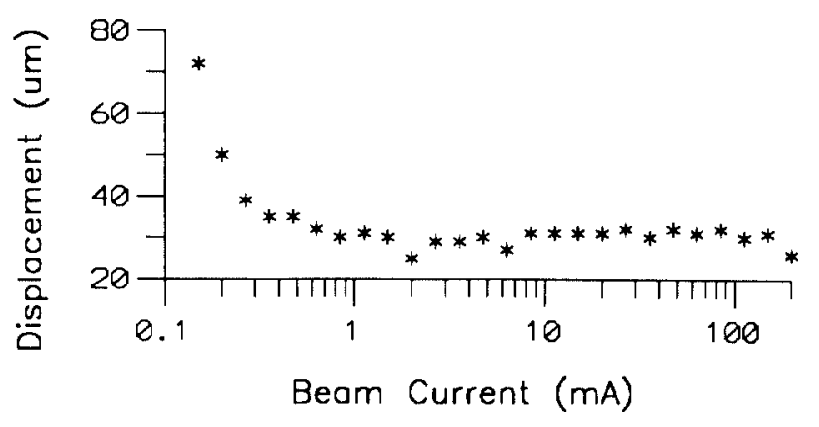

Figure 3. Current dependent drift of the measured beam position.

\section{B. Intensity monitor}

Bergoz's parametric current transformer (PCT) is used to measure average beam current with resolution better than 1 $\mu \mathrm{A}$ ( 1 sec integration window). The output of PCT are digitized by 16 Bits ADC module on VME crate at current stage. The $A D C$ card will be changed to high resolution DVM for high precision measurement. High frequency components of beam current are observed by a fast current transformer (FCT) with rise time better then 1 ns. The beam filling structure at injection phase and routine operation can be observed by FCT.

\section{Stripline electrodes and excitation electrodes}

Two linear tapered stripline [4] electrodes were used to measure transverse motion and time structure as shown in Figure 4 (a). The linear tapered preserve wide bandwidth feature with slightly large amplitude ripple than ideal exponential taper ( $6 \mathrm{~dB}$ from $300 \mathrm{MHz}$ to $6 \mathrm{GHz}$ ) [5]. Easy fabrication was the main reason to choose linear tapered stripline from mechanical point of view. Typical beam response of the linear tapered stripline measured by using 20 $\mathrm{GHz}$ sampling oscilloscope are shown in Figure 4 (b), the bunch length less than 100 psec (FWHM) are observed whell the gap voltage of RF cavity is $300 \mathrm{kV}$.

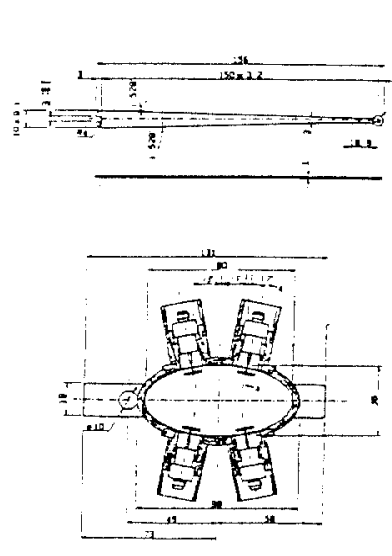

(a)
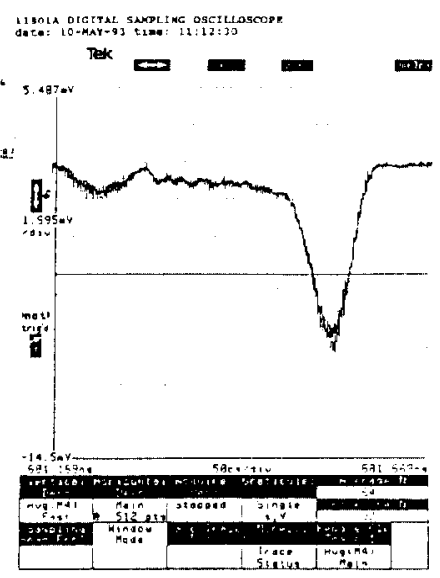

(b)
Figure 4. (a) Sturcture of the linear tapered stripline, (b) Beam response of the linear tapered stripline.
One set excitation electrodes which is composed by four electrodes mounted on skew position of circular vacuum chamber as a magnetic kicker. The excitation electrode is used to excite transverse beam motion or to damp transverse instability if necessary.

\section{Tune measurement}

Two methods are adopted to measure betatron tune currently. One is using RF knockout technique by using the combination of stripline electrodes, excitation electrodes and spectrum analyzer/tracking generator. The alternate approach are using one of the kickers to provide about 1 mrad kick angle, and Fourier analysis turn-by-turn beam position motion by oscilloscope as shown in Figure 5. The synchrotron turn are observed from the synchrotron sideband near the harmonics of the revolution frequency.

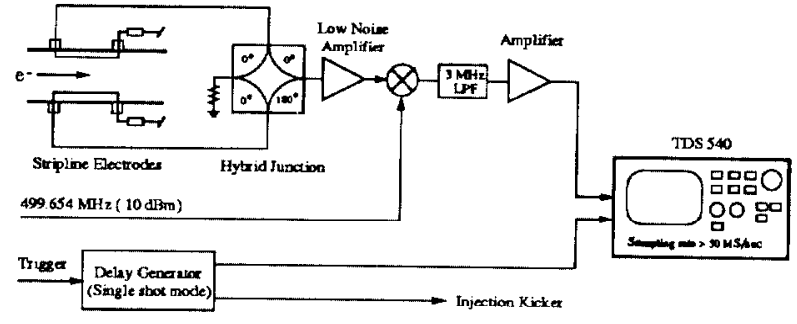

(a)

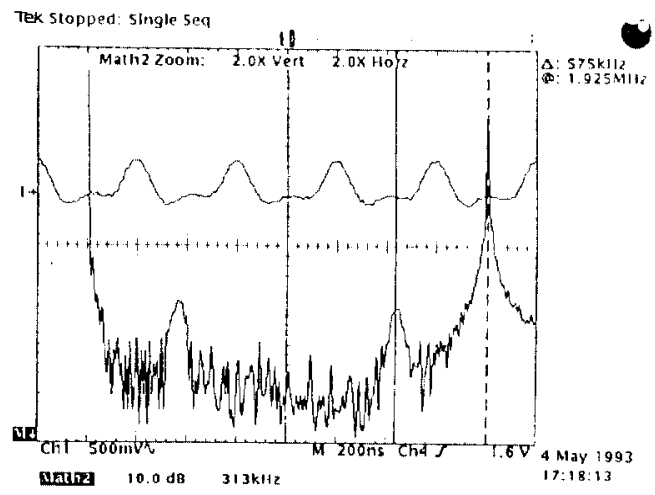

(b)

Figure 5. (a) Betatron tune measurement setup, (b) Typical spectrum of the betatron oscillation.

\section{E. Screen monitor}

Injection conditions and first turn behaviours of the storage ring are study by the aid of eight fluorescent screcns. The screen is mounted on the linear motion feedthrough driven by stepping motor. $\mathrm{CCD}$ cameras are use to observe fluorescent light.

\section{$F$. Synchrotron radiation monitor}

The synchrotron radiation output from one bending magnet was used to measure the transverse profile by XC- 


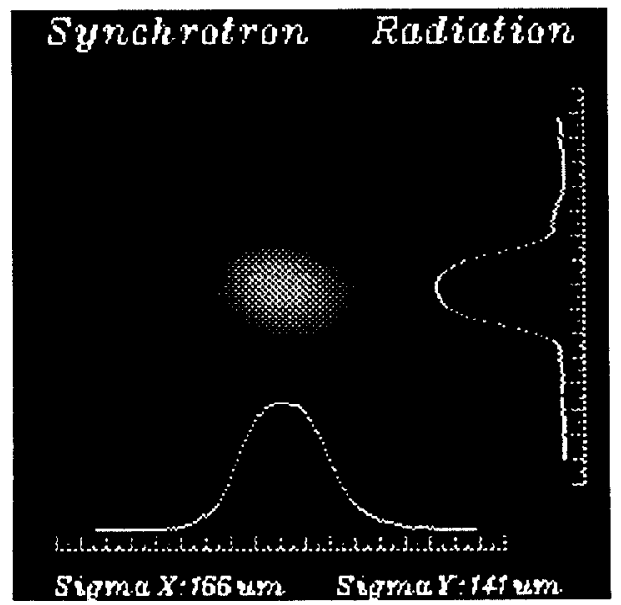

Figure 6. Preliminary result of synchrotron radiation profile measurement, no correction on depth of field and diffraction effect.

77RR high resolution CCD camera. The video signal are captured by frame grabber to extract the transverse profile information. Figure 6 are the the preliminary results of profile measurement. Line scan CCD camera, quadrature diode array, position sensitive detector will be used to measure beam motion. Optical sampling oscilloscope with resolution about $10 \mathrm{psec}$ will be used to measure averaged bunch length.

\section{TRANSPORT LINE DIAGNOSTICS}

The diagnostic devices of the transport line consist of beam position monitors, current monitors, screen monitors and secondary emission monitor. Seven stripline beam position monitors are used to measure electron beam position. The accuracy of the BPM is better than $\pm 0.5 \mathrm{~mm}$. Output of the front-end electronics for BPM are shown in Figure 7. The transmission efficiency of the transport line are measured by Bergoz's fast current transformer as shown in Figure 8.

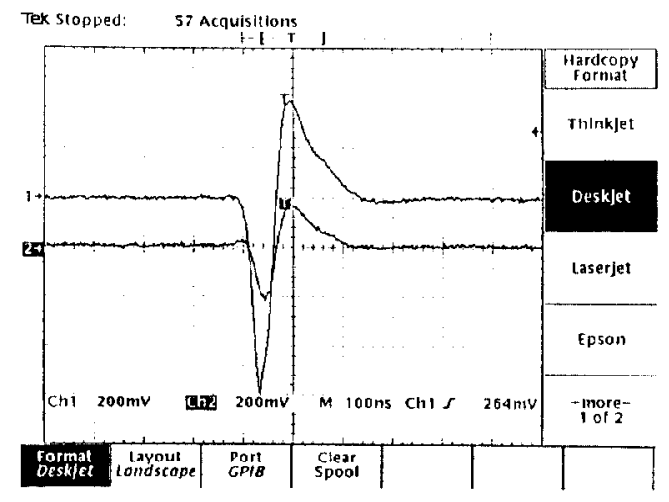

Figure 7. BPM fornt-end electronics output, upper tarce is the response of the left electrode, the lower trace is the right electrode response.

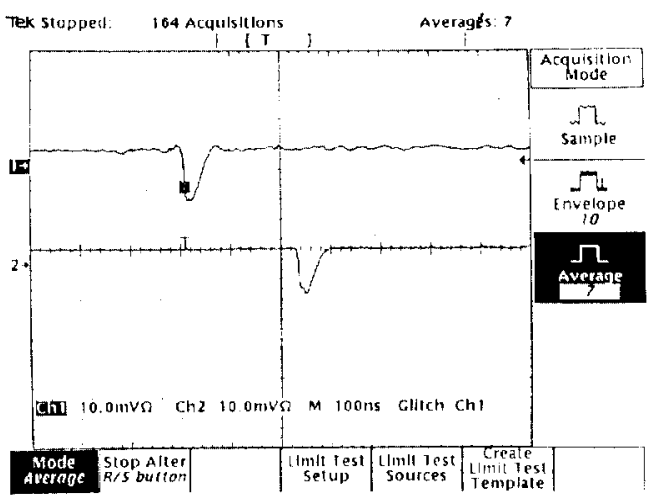

Figure 8. Beam response of the FCT, upper tarce is the response of FCT which is located at upstream of the trasnport line, the lower trace is the doenstream FCT response.

Transverse beam profile and spatial position are observed by six screen monitors. The design of the screen monitor are same as the storage ring. One secondary emission monitor will be used to measured transverse profile.

\section{CONCLUTION}

The commissioning of the storage ring is under way. From the preliminary beam test results shown that most of the diagnostic devices are work functionally as the design specifications. Improvement and reinforcement of the diagnostics system will be continued.

\section{ACKNOWLEDGEMENT}

We are thankful to T. Katsura (KEK-PF), J. Hinkson (ALS), F. Loyer (ESRF), T. Linnecar (CERN) for their helpful suggestion.

\section{REFERENCE}

[1] "SRRC Design Handbook", Synchrotron Radiation Research Center, 1989.

[2] G. J. Jan, and K. T. Hsu, "Beam diagnostic instrumentation of the synchrotron radiation research center", Proc. of the Workshop on Advanced Beam Instrumentation, KEK, Tsukuba , pp.60, April 22, 1991.

[3] G. J. Jan and K. T. Hsu, "Beam Position Measurement System for SRRC", IEEE Proc. of the Part. Accel. Conf. San Francisco, 1157 (1991).

[4] Private communication with J. Hinkson of ALS and T. Linnecar of CERN-SL.

[5] T. Linnecar, "The High Frequency Logitudinal and Transverse Pickup Used in the SPS", CERN-SPS /ARF/78-17, (1978). 